Article

\title{
Amalgamation of Export with Import Information: The Economic Complexity Index as a Coherent Driver of Sustainability
}

\author{
Benjamin Cakir ${ }^{1}$, Isabelle Schluep ${ }^{2}$, Philipp Aerni ${ }^{2}$ and Isa Cakir ${ }^{2, *}$ \\ 1 Institute of Mathematics, University of Zurich, Winterthurerstrasse 190, 8057 Zurich, Switzerland; \\ benjamin.cakir@uzh.ch \\ 2 Center for Corporate Responsibility and Sustainability CCRS, University of Zurich, Zähringerstrasse 24, \\ 8001 Zurich, Switzerland; isabelle.schluep@ccrs.uzh.ch (I.S.); philipp.aerni@ccrs.uzh.ch (P.A.) \\ * Correspondence: to: isa.cakir@ccrs.uzh.ch; Tel.: +41-44-634-59-27
}

Citation: Cakir, B.; Schluep, I.; Aerni, P.; Cakir, I. Amalgamation of Export with Import Information: The Economic Complexity Index as a Coherent Driver of Sustainability. Sustainability 2021, 13, 2049. https:// doi.org/10.3390/su13042049

Academic Editor: Anna Visvizi

Received: 18 December 2020

Accepted: 6 February 2021

Published: 14 February 2021

Publisher's Note: MDPI stays neutral with regard to jurisdictional claims in published maps and institutional affiliations.

Copyright: (c) 2021 by the authors. Licensee MDPI, Basel, Switzerland. This article is an open access article distributed under the terms and conditions of the Creative Commons Attribution (CC BY) license (https:// creativecommons.org/licenses/by/ $4.0 /)$.

\begin{abstract}
Countries that achieve economic complexity in a holistic way are well-prepared to respond to external shocks through internal processes that may also improve their resilience. This article suggests that the Economic Complexity Index (ECI) can capture this 'resilience dimension' of complex economies and assesses their contribution to sustainable change through the amalgamation of export and import information. This novel methodological approach incorporates import information by applying amalgamation on a pre S-Level, which is based on the Lie-Trotter methodology, inducing a Random Walk on a Graph. In the empirical part, this procedure is examined. It shows that the ECI ranking may not always reflect the underlying internal economic complexity of a country, and with it, the country's resilience and contribution to sustainable change. The novel approach is to some extent comparable with the degree of eligibility criteria of the original ECI and consistent with the organic evolutionary character of complex economies. After translating the ECI framework into its stochastic counterpart, the proofs of its interpretation in statistic and probabilistic terms, and its relationship to the Shannon Entropy are conducted. Coherency conditions of sustainability as further eligibility criteria are formulated and the degree of coherency of the ECI is investigated. In view of the challenges related to data preparation, we suggest applying the approach to a broader set of data including import information in order to gain additional insights in a country's internal economic complexity and resilience.
\end{abstract}

Keywords: amalgamation of export with import; coherency conditions; economic complexity; economic performance equivalent; eligibility; internal economic complexity; lie-trotter approach; normalization; random walk on a graph; Shannon entropy; sustainability

\section{Introduction}

The Economic Complexity framework was developed by César Hidalgo and Ricardo Hausmann to measure the accumulated productive knowhow of a country and to deduce from it its future growth prospects [1]. It is based on the assumption that the extent and diversity of human capabilities in a country can be inferred from export data taking into account the diverse range of exported products (diversification) and how broadly they are supplied into world markets (which is expressed by a function of ubiquity) by various countries. In this context, a product is a carrier of information that signals the existence of the requisite capabilities of a country. The focus on the diversification and ubiquity of a product gives insights about the product range and the capabilities in a country, without making any assumptions regarding the nature of these capabilities [1].

Relying primarily on published national export data, Hidalgo and Hausmann [1] constructed the so-called Economic Complexity Index (ECI) on which the Atlas of Economic Complexity is based upon [2]. Compared to the Heckscher-Ohlin model in neoclassical 
economics (including the subsequent more elaborated models that build on it) that relies on a country's factor endowment (labor/capital) to predict its competitive advantage in international trade [3], the ECI represents a major step forward by not just looking at the quantity but also the diversity of exports and ubiquity of the exported products [1].

However, in what sense is the ECI not just related to long-term economic growth, but also to more inclusive and sustainable change? In this paper, we hypothesize that this question can be answered to some extent through a better understanding of the internal complexity of an economy, and its capacity to respond to external shocks through swift shifts in the economy.

\section{Literature Review}

A high degree of internal complexity in an economy is characterized by a large number of specialized firms that operate in networks that contain a large amount of knowledge and knowhow [4]. They have persisted for a long time in tandem with the larger economic ecosystem containing a complementary mix of large, export-oriented firms and resilient domestic companies combined with supportive not-for-profit organizations and public sector institutions, including responsive education and research systems [5]. The agile and innovative companies within this ecosystem that are mostly suppliers of larger exportoriented firms observe trends abroad and then identify a growing dependence on particular imports with no viable counterparts in the domestic economy. They seize the opportunity by making use of their knowledge base, network, and experience to create a domestic product that matches the quality and price of the imported product and then gradually improve it [6-8]. The product may then reach a stage in which it even becomes attractive to be exported, be it due to its superiority in quality, its resource efficiency, or other positive side effects for society and the environment (e.g., produced with less harmful substances under better working conditions) [5].

The firms that produce such products may not yet appear in official export statistics because they are still in the stage of capturing the opportunities in the domestic market first. However, they increase the long-term competitiveness of the country by creating valueadded products that may meet a growing demand abroad as well because they are not just superior in quality but also in terms of sustainability. However, since they operate in a business environment that is characterized by a high content of knowledge and knowhow, human capital may become increasingly scarce and expensive. As a result, new actors in the economic ecosystem emerge to address this scarcity by developing technologies (e.g., robotics) [9] and services (i.e., job matchmaking services) [10] that help lower the search costs for recruiting talent. This then allows to generate higher returns through the adoption and creation of innovation to make up for the high variable costs of labor. They are also more versatile in the sense that they need to respond quickly to new opportunities by customizing their business. This often leads to innovation designed to improve the satisfaction of customers, which then turns out to be of interest to other customers in other market segments [5].

Another advantage of a domestic economy containing a large network of agile smaller firms that cater to the domestic as well as the export-oriented industry is the resulting favorable business environment for the creation of new firms. After all, it is not just a favorable institutional environment and a good public education system that help to lower the costs of doing business. Even more crucial are the availability, timely delivery and quality of existing goods and services, as well as the human capital; all produced primarily for and by the domestic economy. A new firm is much more likely to succeed in a country that has such responsive domestic markets and business services [5].

This favorable business environment is non-tradeable by its very nature and relies to a great extent on social capital that provides the glue that enables firms as well as networks of firms to hold and continuously update knowledge and knowhow [11]. All these aspects combined increase the capacity to replace economically relevant imports, to 
diversify exports, and to strengthen the overall quality and sustainability of the resulting value-added products.

The idea to measure internal complexity of countries is not new. There have been case studies that extended the concept of economic complexity to internal complexity within a country (see e.g., $[7,8]$ ). The results show that using the complexity framework within a country gives important and additional insight about the ability to innovate in a particular domestic industry. Interesting findings of the study conducted by Brummitt et al. [12] show that for the sustainable improvement of an economy, it might be more advisable to have a reliable and gradual increase in technological complexity compared to a premature jump supported by "big push" policies; a finding that may play a relevant role for emerging economies and provide an additional argument to look at the importance of the internal complexity of an economy, in order to have a more qualified view about the future competitiveness and resilience of a country.

\section{Amalgamation Based on the Lie-Trotter Approach to Capture Complexity in a Holistic Way}

The comparability of countries based on different internal data sources and standards in different countries remains a challenging issue. This makes a holistic comparable complexity ranking, including the internal complexity, difficult. However, due to a country's import mix as supplementary information to export data, we can gain significant insights about the country's economy. Namely, we get to know the products that are required in production as well as in consumption. It is obvious that products that are not imported must be produced internally. This provides important additional insights about a country's knowhow that is directly related to its internal economy. We suggest closing this gap by amalgamation that is based on the Lie-Trotter approach on a pre S-Level (see e.g., original paper of Trotter [13] or [14]) of global import information with export information. This merging method offers the possibility for a holistic complexity ranking and accounts for the internal complexity of the economy with respect to the organic evolution thereof.

\section{Main Argument and Hypothesis}

In this paper, we argue that the ECI is not just related to long-term economic growth. However, we also argue that this framework has also a potential to capture the sustainable change and resilience of countries with regard to their ability to mitigate large shocks. In this context, we propose a novel method to better understand how the internal complexity of an economy and its capacity to respond to external shocks through innovative shifts in the economy may improve its resilience and lead to products with more positive external effects for society and the environment.

To achieve this, we suggest the amalgamation of export with import data on a pre SLevel. This would improve the understanding of the economic complexity as a systematic driver of sustainability. At the same time, it would allow to incorporate the internal economic complexity of a country into a comparative assessment and respect the organic evolutionary character of an economy.

\section{Methodological Approach}

In order to be sure that our suggestion fits into a solid theoretical fundament, at the beginning we had to understand the mechanics of the ECI, which are based on the second eigenvector $e_{2}$ (eigenvector associated to second largest eigenvalue in absolute value). For that purpose, we first established the theoretical groundwork with the aim to ensure tractability of our suggestion which we summarize as follows: We first embed the ECI framework into its stochastic counterpart in Section 7.1. Afterwards, in Section 7.2, we interpret the ECI ranking from a statistical, probabilistic, and an information theoretical point of view. For the rigorous mathematical treatment of the mentioned interpretations, we refer to Lemmas 4.1,4.2, 4.3, and 4.5 in the supplementary material. Knowing that the Shannon Entropy represents the expected amount of information transported by identifying the outcome of a random trial on the one hand, and that there exists a certain relationships 
between Shannon Entropy and Kolmogorov Complexity (see comments at the end of Section S4 in the supplementary manuscript based on Theorem 148 in [15]) on the other hand, taken together with our finding for the relationship of the ECI with the Shannon Entropy, this underlines the notion of complexity in the ECI ranking in a certain sense.

Our main results are summarized in Section 8, which is organized as follows: To understand the ECI as a systematic driver of sustainability ranking, we first introduce coherency conditions and summarize the behavior of the ECI as well as alternative rankings with respect to coherency conditions in a standalone (before amalgamation) as well as in amalgamated cases in Section 8.1. The details of the mathematical treatment can be found in Section S5 of the supplementary materials. Roughly speaking, coherency conditions reflect the expected properties of the complexity ranking as a systematic driver of sustainability, which is an additional quality check that is also related to the predictive power of $e_{2}$. In Section S5 of the supplementary material, these investigations are mainly based on the prototype information matrices. However, we also introduce a lemma, which ensures that if the products of the countries are well ordered with respect to the inclusion relation, then $e_{2}$ behaves coherently (see Lemma 5.1 in the supplementary material).

In Section 8.2, we introduce our suggestion of the pre S-Level amalgamation first as a limited process, which intuitively indicates that it is an organic merging methodology followed by a simple formula for computation. The details and mathematical treatments of Section 8.2 can be found in Section S6 of the supplementary material. In Section 9 of this paper, we discuss how our main findings close a relevant research gap. Namely, the incorporation of information about the internal complexity of countries in the ECI framework by means of amalgamation of import with export information on a pre SLevel. This fascinating area offers interesting research ideas, some of which are discussed in Section 10, outlook. Section 11 provides concluding remarks, which mainly relate to aspects like the eligibility conditions and their tradeoffs, which we managed to take into account to a certain degree.

\section{Empirical Validation Which Motivates the Suggested Approach}

In this section, we discuss the need to merge supplementary information such as import data with export information. Thereby, we scrutinize key economic sustainability indicators of selected countries and demonstrate that the ECI ranking not always reflects the underlying internal economic complexity of a country and raises questions as to the necessity for enhancement.

Inspired by the matrix operations governing the dynamics of commutative systems, we used the so-called Lie-Trotter product formula, which we think is also appropriate for non-commutative interacting systems with an organic evolutionary character. According to our analyses, the amalgamated process seems to "favor" a balanced import (complexity)/export (complexity) ratio. Keeping this in mind, we have selected countries that we think have a too low ECI ranking from a sustainability point of view when the ECI is based on export data only; and where we think that enhancing the ECI by amalgamation of export and import data might add additional insight and improve the quality of the ECI ranking when taking sustainability into account.

With the aim to account for the different degrees of internal complexity, we compare in this section the economies of New Zealand and Singapore, among others. While New Zealand represents a country that makes use of the global knowledge economy to develop and eventually export value-added agricultural products and has a low ECI ranking, Singapore is a global trading hub for many different products and services with a high ECI ranking. By means of these two countries, we intend to demonstrate that the apparent gap in the ECI ranking between the two countries could be closed by taking into account the actual degree of internal complexity by means of amalgamation of export with import data.

Tables 1 and 2 display key indicators of selected countries that have on the one hand very high and on the other hand relatively low ECI rankings. Table 1 illustrates that 
independent of the ECI ranking, the selected countries show similar per capita values regarding imports and exports of goods and services and gross value added (GVA).

Table 1. Comparison of imports, exports, and gross value added per capita among high and relatively low Economic Complexity Index (ECI) performing countries.

\begin{tabular}{|c|c|c|c|c|}
\hline Country & $\begin{array}{c}\text { ECI Rank (Average } \\
\text { 2014-2018) }\end{array}$ & $\begin{array}{l}\text { Import of Goods and } \\
\text { Services (Constant } \\
2010 \text { US\$) Per Capita }{ }^{2}\end{array}$ & $\begin{array}{l}\text { Export of Goods and } \\
\text { Services (Constant } \\
2010 \text { US\$) Per Capita }{ }^{2}\end{array}$ & $\begin{array}{c}\text { Gross Value Added at Basic } \\
\text { Prices (GVA) (Constant } 2010 \\
\text { US\$) Per Capita }{ }^{2}\end{array}$ \\
\hline New Zealand & 52 & 12,084 & 11,409 & 34,504 \\
\hline Australia & 88 & 12,459 & 12,962 & 52,508 \\
\hline Canada & 38 & 16,435 & 16,199 & 48,049 \\
\hline Japan & 1 & 7764 & 8067 & 33,584 \\
\hline Switzerland & 2 & 44,516 & 55,314 & 54,715 \\
\hline Germany & 3 & 20,269 & 23,110 & 28,875 \\
\hline Singapore & 6 & 96,558 & 113,763 & 40,049 \\
\hline South Korea & 4 & 13,387 & 14,317 & 15,244 \\
\hline
\end{tabular}

Sources: ${ }^{1}$ Atlas of Economic Complexity [16]; ${ }^{2}$ calculated from World Bank Development Indicators [17].

Table 2. Comparison of GDP, unemployment, and wealth among high and relatively low ECI performing countries.

\begin{tabular}{ccccc}
\hline Country & $\begin{array}{c}\text { ECI Rank (Average } \\
\text { 2014-2018) }\end{array}$ & $\begin{array}{c}\text { 1 GDP Per Capita, PPP } \\
\text { (Current International } \\
\text { \$), Average 2015-2019 } \mathbf{~}^{2}\end{array}$ & $\begin{array}{c}\text { Unemployment, Total (\% of } \\
\text { Total Labor Force) } \\
\text { (Modeled ILO Estimate), } \\
\text { Average 2015-2019 2 }\end{array}$ & $\begin{array}{c}\text { Median Wealth 2019 } \\
\text { (US\$ Per Adult) }\end{array}$ \\
\hline New Zealand & 52 & 41,155 & 4.7 & 116,437 \\
Australia & 88 & 49,378 & 5.6 & 181,361 \\
Canada & 38 & 48,241 & 6.3 & 107,004 \\
\hline Japan & 1 & 41,159 & 2.8 & 110,408 \\
Switzerland & 2 & 67,429 & 4.8 & 227,891 \\
Germany & 3 & 52,353 & 3.8 & 35,313 \\
Singapore & 6 & 94,545 & 4.0 & 96,967 \\
South Korea & 4 & 40,728 & 3.8 & 72,198 \\
\hline
\end{tabular}

Sources: ${ }^{1}$ Atlas of Economic Complexity [16]; ${ }^{2}$ World Bank Development Indicators [17]; ${ }^{3}$ Credit Suisse Global Wealth Databook 2019 [18].

Table 2 further demonstrates that the gross domestic product (GDP) per capita is similar among countries with high and relatively low ECI rankings. The unemployment rate in two of the countries with lower ECI ranking is higher than the rest, but not substantially. Median wealth per adult is on average even higher in countries with lower ECI ranking than in the high ECI ranking countries.

On the basis of the key economic indicators of countries in Tables 1 and 2 we can determine e.g., the average GDP per capita in percent of import of these two classes of countries (which is about 340\% for New Zealand, Australia, and Canada on average, compared to about 200\% for Japan, Switzerland, Germany, South Korea, or Singapore, on average). Based on this, we cannot explain why the ECI ranking of New Zealand, Australia, and Canada is that far off from the top ECI ranking countries Japan, Switzerland, Germany, South Korea, or Singapore. For example, a closer look at New Zealand's export and import mix suggests that the country has found a sustainable balance in transforming the strength gained from export of agricultural products into an internal economy that can be justified by its comparably low unemployment rate and GDP per capita. As a champion of some agricultural export products, New Zealand seems to own unique knowhow that is supplemented by its natural resources. The country optimizes its economic options with efficient technological support through balanced imports, and the latter gives it the ability to manage extreme economic crises.

On the other hand, by looking only at the unemployment rate and GVA per capita of Singapore, we cannot reject the idea that Singapore's economic sustainability ranking 
should be lower than its ECI ranking. On the other hand, when considering the average GDP per capita for the top ranked countries in percent of their imports, which is about $200 \%$, we notice that the average for Singapore is significantly lower with less than $100 \%$. Singapore seems to be a special case compared to the other top ECI ranking countries. Based on these facts, one might think that Singapore is a counterexample to our hypothesis. However, the question is, "what is the origin of this strength"? Is it the accumulation of productive knowhow within the country or is it its trading skills or other conditions? The strength of Singapore most probably is related to the country's ability to crate favorable economic framework conditions and its interconnectedness and is therefore different from the accumulated productive knowhow. In fact, the very large network of free trade agreements (FTA) gives Singapore widespread access to international markets at preferential conditions. Although at this stage we are not in a position to question the sustainability of Singapore, we observe that the import content of exports of Singapore is in the top 10 according to OECD data. This indicates to a certain extent that the accumulated productive knowhow of Singapore is different from the top five ECI countries. Therefore, in the case of Singapore, we rather argue that amalgamation of import data might slightly downgrade the ECI of Singapore. However, FTAs as an additional supplementary information could slightly upgrade it if it is merged. This could rather lead to a different interpretation than "accumulated knowhow" of ECI.

As a summary of above observations, New Zeeland on the one hand and Singapore on the other hand, we can conclude that amalgamation of import information to the ECI framework has a good chance to increase its quality as an indicator for measurement of the accumulated productive know how. New Zealand supports the hypotheses that some countries could be upgraded in the ECI ranking if taking imports into account. Of course, the updated (export and import data) ECI ranking can be merged further with additional network information such as the number of FTAs for a more complete measurement of economic sustainability. This would be a different message than what we understand under the current ECI ranking. In this sense, Singapore does not represent a counterexample to our hypothesis.

\section{Interpreting the Building Blocks of the ECI in Stochastic Terms}

Our main goal is to suggest a model that can merge supplementary information sources, which develop organically. Therefore, it makes sense to interpret the building blocks of the ECI in stochastic terms. We proceed as follows: First, the method of reflection has to be embedded in matrix language followed by its stochastic counterpart; second, the second eigenvector $e_{2}$ has to be interpreted in statistic, probabilistic, and information theoretical terms, and the relationship of $e_{2}$ with the Shannon Entropy has to be confirmed; third, a framework has to be established for the engineering of the matrix $S$ and an economic performance equivalent (EPE) has to be introduced as a version of import to export so that they are compatible in order to perform the amalgamation operation; fourth, the coherency conditions of ranking with respect to sustainability have to be established in order to assess the quality of our suggestion.

A main finding is that the Random Walk on a Graph generated by amalgamation of export with import information on pre S-Level by means of the Lie-Trotter formula has the desired properties. For the quality of our suggestion, we conducted the following steps: First, we confirmed the symmetry and positivity of the amalgamated matrix S; second, we formulated conditions of coherency and of a sustainability index; third, we investigated the coherency behavior of the second eigenvector $e_{2}$ in the standalone case and compared it with a Random Walk on a Graph based on amalgamation of export with import information on a pre $S$ level. The mathematical treatments as well as the numerical analyses in the above-described procedure can be found in the manuscript, which is provided in the supplementary materials. 


\subsection{Embedding the Method of Reflection in Matrix Language and Its Stochastic Counterpart}

In general, given a trade information matrix (export or import) $Y$ of a closed system of countries, we can construct a symmetric matrix $S$ weighting the "connectivity" of the countries. An important example of this process was introduced by Hidalgo at al. where they introduced zero one matrix information by putting $Y(i, j)=M(i, j)=1$ if country $i$ exports the product $j$ and zero otherwise [1]. Based on $M$, two kind of weights with respect to countries resp. products can be constructed:

$$
\begin{aligned}
& S_{C}=M \times D_{1} \times M^{t r} \\
& S_{P}=M^{t r} \times D_{2} \times M
\end{aligned}
$$

here, $D_{1}$ and $D_{2}$ are diagonal matrices. Their diagonal elements are defined by:

$$
D_{1}(j, j)=\frac{1}{\text { number of countries exporting } j}
$$

and

$$
D_{2}(i, i)=\frac{1}{\text { number of products exported by } i}
$$

which are the reciprocal of ubiquity of exported products and the diversity of the countries, respectively. Due to analogy, in the rest of this paper we only focus on $S_{C}$ and write $S$ in order to avoid notational overflow.

The element of matrix $S(i, i)$ of $S$ can be interpreted as expected common knowledge shared by $i$ and $i$ (see supplementary material Section S3.4 for more insight). The normalization of its rows delivers transition probability matrix $P$ governing the associated Random Walk on a Graph:

$$
P=D \times S
$$

If we denote the paths of a Random Walk on a Graph with an initial distribution $\mu$ with $\omega_{t}^{\mu}$, we can define a stochastic process $X_{t}^{\mu}$ with paths $X\left(\omega_{t}^{\mu}\right)$ for any bet (or payoff function) $X$ defined on the static state space of the Random Walk (which can be identified by the initial knowhow state of the countries).

\subsection{Interpretations of $e_{2}$}

The propagator $P$ can be used to determine the expected payoff of $X_{t}^{i}$ with an initial state $i$ :

$$
u(t)=P^{t} \times X
$$

The elements $u(t)_{i}$ : of the time dependent column vector $u(t)_{i}$ result exactly in the expected payoff of the process $X_{t}^{i}$, which reads as expected payoff of $X_{t}$ given the initial state $i$ : Under mild assumptions, excluding the degenerate cases (a) non-zero contribution of $e_{2}$ in eigenvector decomposition of $X$ and (b) the strict dominance of second eigenvalue $e_{2}$ comparing to other eigenvalues excepts, of course the first eigenvalue, we can prove the following statements:

Lemma 1. There exist a $t_{0}$ so that $R(u(t))=R\left(e_{2}\right)$ for all $t>t_{0}$ with an adequate choice of sign for $e_{2}$.

Here, $R$ is the ranking function defined on the set of all elements in $\mathbf{R}^{m}$, which have pairwise different components.

Lemma 2. There exist $a t_{o}>0$ so that the rank of the transition probabilities of switching from one similarity class two others at a future time $t$ is given by the rank of $e_{2}$ for all $t>t_{0}$.

Intuitively, Lemma 2 can be interpreted as: "better" ones in a "superior" class and "worse" ones in an "inferior" class are likely to stay longer in their class. In this context, 
we assume two similar classes that are defined by the sign of the components of $e_{2}$. The first statement (Lemma 1) together with the special properties of logarithm imply the next statement from an information theoretical point of view and justifies the notion of "complexity".

Lemma 3. If we exclude "pathological" cases, then there exist a $t_{0}$ so that the rank of the Shannon Entropy of the conditional probabilities $P^{t}(i,$.$) is given by the rank of e_{2}$ for all $t>t_{0}$ if the sign is adequately chosen.

Here, the Shannon Entropy (see [19] for further readings on Shannon Entropy) of the conditional probabilities $P^{t}(i,$.$) is defined by$

Finally, with the average of the terms $\mathrm{H}(s,$.$) for s \leq t$ we gain another alternative:

$$
A H(t, i)=\frac{\sum_{s \leq t} H(s, .)}{t}
$$

Lemma 4. There exist a $t_{0}$ so that, the rank $A H(t,$.$) is constant for all t>t_{0}$.

In this sense, our numerical tests support the ranking with $\mathrm{AH}(t,$.$) with large enough$ $t$ is a more coherent method with respect to diversity and ubiquity also after the amalgamation of import information.

A rigorous treatment of the above statements can be found in Section S4 of the supplementary manuscript (see Lemmas 4.1,4.2, 4.3, and 4.5, respectively). The results are general and would work not only for Random Walks on a Graph based on zero one information matrices, but also for other Random Walks on a Graph based on real, valued information matrices.

\subsection{Engineering of the Weight Matrix $S$ and the Economic Performance Equivalent (EPE) of Import}

There exists a vast amount of literature that suggests improving the ECI based on export information alone [20]. As far as we can assess, almost all approaches are based on the ranking of the second eigenvector $e_{2}$, but with different approaches for the engineering of $S$. In order to have certainty that our suggestion of amalgamation of import with export information adds value to the quality of a complexity and sustainability raking, the following question was relevant for us: Can it be that one of these approaches implicitly covers our suggestion?

We answered this question with a mindset based on generic theoretical weight matrix $S$, where its elements $S(i, i)$ can be interpreted as expected value of common knowledge shared by $i$ and $i$ (see Sections S3.3 and S3.4 in the supplementary material). For its construction, we assume three prototype behaviors (indicating the dependency behavior of the market players) at the micro level, driving the evolution of complexity in order to challenge the potential of our suggestion.

The generic weight matrix $S$ takes the following extreme prototype dependency behavior of market players into account in order to identify common knowledge of the countries: Absolute independent behavior; absolute dependent behavior; and complementary behavior of market players. The reality will be between these three extreme prototypes (e.g., convex combinations) on product level and weight of the above prototypes depend among others on the other trade information driving the fragments of common knowledge between the countries. For details we refer to Section S3.4.2 in the supplementary material.

Dopfer et al. suggest that an evolutionary agent takes external as well as internal structures in his engagement of solving problems into account [21]. Our conclusion is that supplementary import information adds value to quality in order to describe the evolution of knowhow. In other words, the estimation of expected common knowledge, which is based only on export information, leaves out the common knowledge, which can result 
from the internal economy. However, export together with the supplementary import information deliver additional insights about the fragments of common knowledge that is based on the internal economy. These theoretical considerations are also supported by Section 6 of the empirical context. As a summary, we can say that merging import with export information is relevant. In Section 4, we answer the question of how the mentioned merging procedure should be conducted in an eligible manner that reflects the organic character of an economy.

\section{Analysis}

\subsection{Coherency of $e_{2}$ as Sustainability Ranking and Other Alternatives}

The statistic, probabilistic, and information theoretical properties of $e_{2}$ are appealing and indicate similarities with respect to complexity except in some rare cases. However, these properties do not answer its relationship with sustainability. Our coherency investigation (with respect to sustainability) gives an acceptable answer to this question. Namely, $e_{2}$ behaves logical (minimal requirements) and accepts some "pathological" cases. However, this happens only to a certain extent with respect to common sense sustainability conditions, and does not fully satisfy the following natural conditions in terms of sensitivities:

(1) If we only increase the diversity of a specific country $i$ without an expense (e.g., discovering a side product without an expense), then $i$ should not have less favorable ranking then before comparing individually any other country.

(2) Decrease the ubiquity of a product of the country $i$ by means of waiving the same product by another country, then $i$ should not have less favorable ranking.

Of course, one can disagree with the above statements as general condition due to special cases in practice, where even a proven causal relationship justifies the opposite. However, we rather think that the justification of this kind of causal relationship proving the opposite of above conditions need an additional information source and a metric based on only export data is rather incapable of delivering an insight on this level.

In the context of the present paper, economic sustainability must be related by definition to the ability to survive or coexist as a unit (countries, cities) in the long run. In other words, a country's sustainability must be understood as 'future ability' ensured by a domestic economic ecosystem that is not just competitive in the classic sense of pricecompetitiveness but through its ability to respond to social and environmental challenges with scalable and therefore commercially viable innovation. Therefore, we believe sustainability management is in a sense a more difficult risk management, because it is not just focused on preventing risks, but also seizes opportunities.

As good news, we note that in case of full comparability of the countries (for any pair of two countries, one of them in the pair has all the products of other country) we have no surprise: With an adequate choice of the sign of $e_{2}$, given comparability of product ranges, the countries with a larger product range dominate the ones with a smaller product range. We have also investigated other alternatives with respect to sustainability conditions. e.g., the diagonal of weight matrix $S$ seems to behave well and also after amalgamation does not induce surprises. Although seemingly natural, rigorous treatment of this finding is tricky (see Lemma 5.1 in the supplementary material).

As a summary, we conclude that $e_{2}$ is an adequate complexity ranking instrument except in some "rare" cases and behaves after amalgamation as good as before amalgamation. However, for a leading economic sustainability index, which also behaves well after amalgamation of supplementary information, a comprehensive investigation could be useful. The diagonal of the weight matrix $S$ is one alternative, which, however, does not have the mentioned elegant interpretations.

8.2. Main Result: Definition and Interpretation of Amalgamation of Random Walks on P-Level as well as on Pre S-Level

Assume that we have a Random Walk on a Graph that is governed by a transition probability matrix $P_{e x}$ and $P_{i m}$, which is generated by export and information, respectively. 
A natural idea would be to mix both matrices by their product $P_{e x} * P_{i m}$ inducing a time inhomogeneous periodic process. However, this idea has its drawback and if $P_{e x}$ and $P_{i m}$ are not commutative, the stationary of the mixture as well as its second eigenvector $e_{2}$ depend on the order of multiplication. However, if we define a matrix operation to be infinite decimally (which can be interpreted as an organic mixture) and power it accordingly, the matrix operation becomes commutative: Put

$$
P_{e x, i m, n}=P_{e x}^{\frac{1}{n}} \times P_{i m}^{\frac{1}{n}}
$$

and define

$$
P_{e x, i m}:=\operatorname{Lim} P_{e x, i m, n}^{n}
$$

The matrix operation above refers to Lie-Trotter product formula, which we call amalgamation on a P-Level in our context. However, although we have satisfactory numerical results, we cannot claim that it is always a Random Walk on Graph with positive eigenvalues. However, the same idea works on pre S-level.

Put

$$
S_{e x, i m, n}=S_{e x}^{\frac{1}{n}} \times S_{i m}^{\frac{1}{n}}
$$

and define

$$
S_{e x, i m}:=\operatorname{Lim} S_{e x, i m, n}^{n}
$$

The $S_{e x, i m}$ defined as above is symmetric, has a positive eigenvalue, and its normalization delivers a Random Walk on a Graph under mild conditions. We call this Random Walk as amalgamated Random Walk on pre S-level (see $[10,11]$ for the details of theoretical treatment of Lie-Trotter product). Our numerical results show that $S_{e x, i m}$ as well as the amalgamated Random Walk behave well and have balancing properties, which means that a country with a superior import profile is likely to be upgraded.

Under mild conditions the above limes exist and can be written as:

$$
S_{e x, i m}=\operatorname{Exp}\left[\log \left(S_{e x}\right)+\log \left(S_{i m}\right)\right]
$$

where $\operatorname{Exp}(\mathrm{A})$ and $\log (\mathrm{A})$ are exponential and the logarithm function of matrix A respectively if they exist (which exists under mild assumptions, see [22] for details). Please note that the above Formula (13) coincides with the usual matrix product if $S_{e x}$ and $S_{i m}$ are commutative.

Let us give some more insight about this merging approach by illustrating the interaction process supporting the organic evolution of the productive knowhow with the following Figure 1.

On a micro level, the knowhow evolution of each producer (or market player) will be impacted by domestic and non-domestic interactions. These interactions happen on a discrete time points and the evolution of the knowhow of the individual producer might very well depend on the order of its interaction with external and internal environment. However, on an aggregated macro level for large enough sample (e.g., producer of a country) it makes sense to assume that these interactions happen continuously. At least locally, this gives rise to assume that up to a time scaling factor, $S_{e x}^{t}$ and $S_{i m}^{t}$ represents evolution of expected values of common knowledge shared by $i$ and $i$, based on the export and economic performance equivalent of import information matrices at time $t$, respectively. For the economic performance equivalent of import information, we refer to Section S3.4.3 of supplementary material, which is closely related to internal demand. The evolution matrices $S_{e x}^{t}$ and $S_{i m}^{t}$ solve meaningful differential growth equations and indicate in some sense exponential information growth. Under these assumptions, as in Figure 1, we can combine the evolution $S_{e x}^{t}$ with $S_{i m}^{t}$ in an interval of length $2 / \mathrm{n}$ by a successive application of the nth root of $S_{e x}$ and $S_{i m}$ :

$$
S_{e x, i m, n}(i, \tilde{\imath})=S_{e x}^{\frac{1}{n}} \times S_{i m}^{\frac{1}{n}}(i, \tilde{\imath})
$$


By taking the nth power of $S_{e x, i m, n}(i, \tilde{\imath})$, we obtain a discrete process that depends on the order of the matrix multiplication in (14). However, for large $n$ the mentioned dependence on the order becomes marginal. Finally, taking the above procedure to limit leads to the mentioned formula for amalgamation of $S_{e x}^{t}$ with $S_{i m}^{t}$ :

$$
\begin{aligned}
S_{e x, i m} & =\operatorname{Lim} S_{e x, i m, n}^{n} \\
& =\operatorname{Exp}\left[\log \left(S_{e x}\right)+\log \left(S_{i m}\right)\right]
\end{aligned}
$$

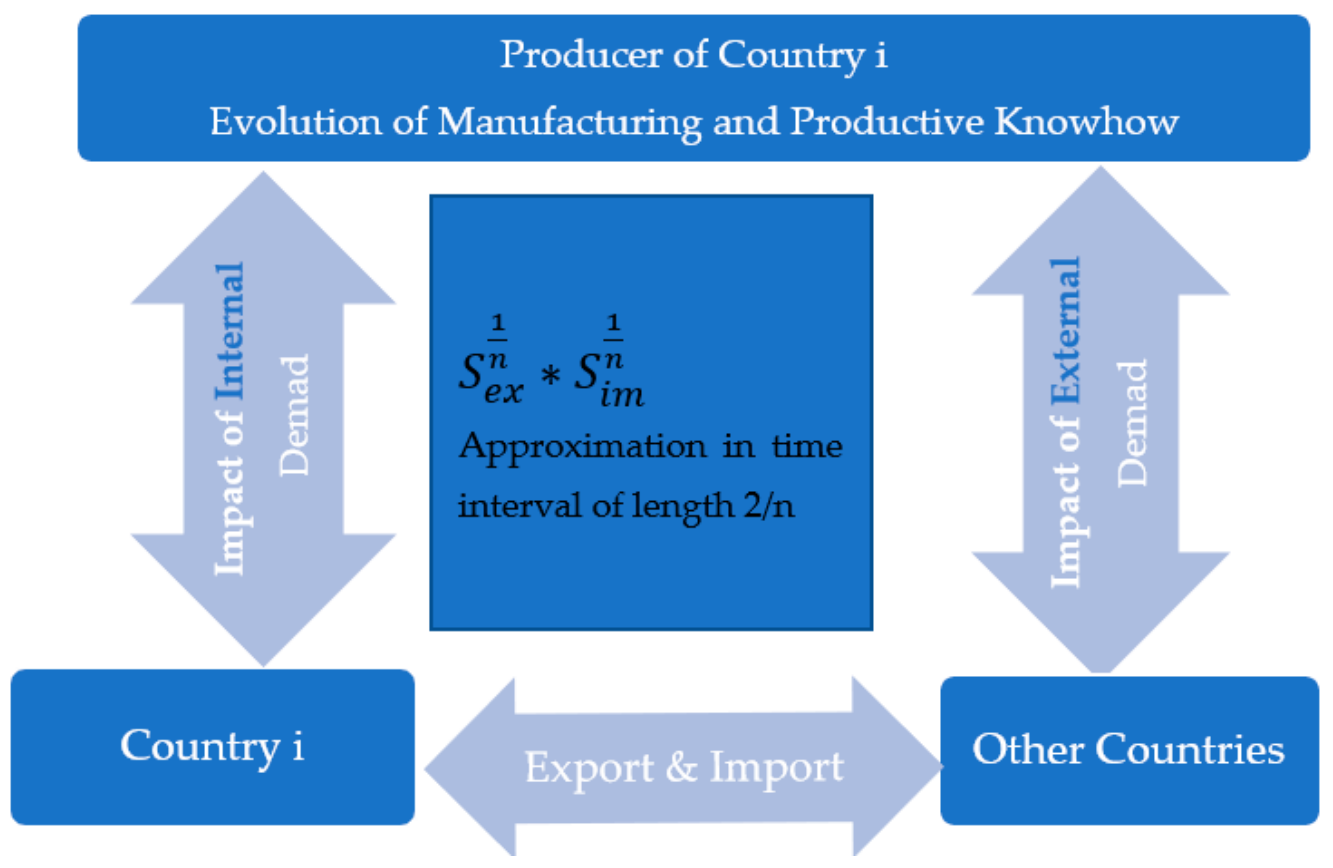

Figure 1. Organic evolution of productive knowhow.

\subsection{Model Risk and Numerical Validation}

One way to compensate the lack of data and to minimize the model risk is to investigate its behavior on prototype examples on predefined eligibility criteria and to compare it with different models. In this sense, we examine the degree of eligibility of the introduced standards for $e_{2}, H(t,$.$) , and \mathrm{AH}(t,$.$) and compare it with the diagonal elements of the$ amalgamated $S_{e x, i m}$, which is eligible with respect to the coherency conditions diversity, ubiquity, and monotony. Table 3 summarizes our numerical tests.

Table 3. Summary results of the numerical tests and theoretical coherency implications.

\begin{tabular}{cccc}
\hline Ranking Method & Consistency with Respect to \\
Equivalent Classes & $\begin{array}{c}\text { Coherency Criteria } \\
\text { (before Amalgamation) }\end{array}$ & Monotony \\
\hline$e_{2}$ & $\begin{array}{c}\text { On prototype examples not } \\
\text { satisfied }\end{array}$ & $\begin{array}{c}\text { To some degree, on some } \\
\text { prototype examples not satisfied }\end{array}$ & $\begin{array}{c}\text { Satisfied by the monotony Lemma } \\
5.1 \text { in supplementary materials }\end{array}$ \\
$H(t,)$. & $\begin{array}{c}\text { Satisfied also on prototype } \\
\text { examples }\end{array}$ & Analogous to $e_{2}$ & Analogous to $e_{2}$ \\
$A H(t,)$. & Analogous to $H(t,)$. & To a large extent satisfied & Analogous to $e_{2}$ \\
Analogous to $H(t,)$. & To a larger extent satisfied & Analogous to $e_{2}$ \\
\hline
\end{tabular}

In some "pathological" cases, countries can be segregated into two classes, where each element of one class has a counterpart in the other class. In such cases, the absolute value 
of the components of $e_{2}$ is coherent. However, then $H(t,$.$) adapts its ranking according to$ the absolute value of the components $e_{2}$ for large enough $t$ automatically.

With the term "monotony" in Table 3, we mean the following condition: If product sets of the countries can be ordered totally with respect to inclusion relations (which means that country 1 has all the exported products of country 2, country 2 has all the exported products of country 3 , country 3 has all the exported products of country $4 \ldots$ and so on) then we expect that any eligible ranking system orders the countries accordingly, which means that the rank of country 1 should be more favorable than rank of country 2, rank of country 2 should be more favorable than rank of country 3 and so on. In this sense, all the above alternatives behave coherently (see Lemmas 5.1, 5.2 and remark 1 in supplementary material). Moreover, if we use the notation $\leq_{C}$ for comparing the degree of coherency between ranking methods, we can summarize our theoretical and numerical investigations as follows:

$$
\begin{aligned}
e_{2} & \leq_{C} H(t, .) \text { for large enough } t \\
& \leq_{C} \mathrm{~A} H(t, .) \text { for large enough } \mathrm{t} \\
& \leq_{C} \text { Diagonal of } \mathrm{S} .
\end{aligned}
$$

We would like to emphasize that due to the Entropy Lemma 3 in Section $7.2 e_{2}$ and $H(t,$.$) for large t$ are up to some special cases equivalent, which could also be confirmed numerically. The ranking methods $e_{2}, H(t,),. \mathrm{AH}(t,$.$) and the diagonal elements of the$ amalgamated $S_{e x, \text { im }}$ behave similarly to a certain extent. However, we are also interested in the balancing impact of amalgamation which is the compensation effect of strong internal economy (given by economic performance equivalent import information matrix). Table 4 summarizes our theoretical and numerical work.

Table 4. Balancing behavior of ranking methods after amalgamation.

\begin{tabular}{cc}
\hline Ranking Method & Balancing Behavior \\
\hline$e_{2}$ & Not satisfied on some prototype examples \\
$H(t,)$. & Up to special prototypes cases analogous to $e_{2}$ \\
$A H(t,)$. & To a large extent satisfied \\
Diagonal of $S$ & To a larger extent satisfied \\
\hline
\end{tabular}

According to our analysis, amalgamation of import with export information has a balancing effect in descending order in the above table. In the amalgamated case, we can give an additional porotype example consisting of monotone decreasing and monotone increasing information matrices, which induces two classes of countries, where each element of one class has an equivalent counterpart in the other class. Similar to the case before amalgamation, also in the case of amalgamation the absolute value of the components of $e_{2}$ is coherent and $H(t,$.$) adapts its ranking according to the absolute value$ of the components $e_{2}$ for large enough $t$ automatically.

Finally, we would like to note that we also conducted simulations of rankings in order to measure the correlations of above ranking methods. It turns out that the correlation of two methods is driven by the degree of coherencies, which is not surprising. For example

$$
\begin{aligned}
\operatorname{Corr}( & \left.R(\text { diagonal of } S), R\left(e_{2}\right)\right) \\
& \leq \operatorname{Corr}(R(\text { diagonal of } S), R(H(t, .)) \\
& \leq \operatorname{Corr}(R(\text { diagonal of } S), \mathrm{R}(\mathrm{AH}(\mathrm{t}, .))
\end{aligned}
$$

In the above expression $R(H(t,)$.$) and R(A H(t,)$.$) means ranking by H(t,)$.$) and$ $A H(t,$.$) for by means of large enough t$. More insight about above summaries can be found in Section S5 of the supplementary material.

\section{Discussion}

Ideally, we would need all the relevant value chain information from the origin to the end for the ranking of accumulated productive knowhow as a significant driver of 
economic sustainability. Even though that does not seem to be a realistic requirement, the assumption serves as a good start with a learning instrument that ranks sustainability in a holistic way. After all, a measurement framework that can learn coherently through the incorporation of supplementary value chain information will be able to continuously improve the quality of output and therefore become more valid over time. We believe that this goal can be achieved in a pragmatic and modular way with our suggestion of amalgamation of import with export information. In this sense, we could give an alternative answer to the following three questions:

(1) Is it necessary to enrich the ECI with supplementary value chain information such as import trade data?

(2) How can we merge supplementary information to improve the quality of the ranking system and avoid non-intended massages and false interpretation as much as possible?

(3) What is the expected contribution of the suggested enhancement?

In this paper, we answer the above questions as follows:

Answer to first question: The empirical validation presented in Section 6 supports the relevancy of supplementary value chain information such as import trade data in order to improve the quality of the ECI.

Answer to the second question: We argue that the amalgamation of import data on the S-level is an adequate approach to close the gap related to the first question. The intuition behind our approach is the imitation of the behavior of market players. The latter is influenced by external as well as internal environments, which successively drive the dynamics of market players in infinite decimal time periods. In this way, we are in line with the organic evolution of the economy, governed by two dynamics (a Random Walk on a Graph induced by amalgamation of two weight matrices $S_{e x}, S_{i m}$ of a graph both resulting from evolutionary processes, see Sections S3.3 and S3.4 of supplementary material) generated by two supplementary information matrices. Our suggestions also satisfy the desired mathematical properties allowing statistical, probabilistic, and information theoretical interpretations. Moreover, we have conducted numerical analysis in order to test the coherency behavior of amalgamation indicating e.g., the preference of balancing of export with import data. Although we could not test our suggestion with real world data, the above work supports our hypothesis that our suggested approach can improve the quality of the ECI as a systematic driver of sustainability. We would also like to note that the balancing impact of our suggested approach depends on the ranking method. For example, the ranking by the Shannon Entropy (which is almost equivalent version of ranking method of ECI indicated by Lemma 3 of Section 7.2) would result in a slightly more sensitive balancing impact than the ranking metrics of ECI. This implies that a favorable economic performance equivalent of the import information matrix compensates for a poor export product profile in our suggested amalgamation approach in a more sensitive way. The details of our methodology, and the conducted numerical analysis, can be found in the supplementary material.

Answer to the third question: We argue that the amalgamation of import data as supplementary value chain information to export data gives insight also into the internal complexity of an economy. It opens the possibility to construct a comparable internal complexity of the countries based on global export and import data. However, this additional output requires further engineering (see Section 10, outlook).

\section{Outlook}

During our research, we had intensive discussions that were of theoretical and practical nature and our investigations became much more comprehensive as we had planned for at the beginning. According to our literature research, we could not find self-contained groundwork with which we could connect the theoretical quality of our main suggestion amalgamation of import the information with the export information on a pre S-Level. However, also due to obvious data challenges, we were not able to conduct comprehensive 
empirical tests. Luckily, our team was a mixture of persons with complementary skills, which was exactly what was needed in order to close these gaps. Our supplementary material is a mixture of theoretical and numerical treatments, which summarizes the workarounds in a self-contained manner for our purposes. At this point, we present the weak points of our findings, how they could be developed further, and how our work could contribute to other interdisciplinary areas. The following aspects we consider the most interesting:

- Coherency conditions of sustainability: During our research for this paper, we felt a strong necessity to formulate coherency conditions of sustainability, because a broad consensus for its specification is required. In this sense, we have suggested a list of coherency conditions reflecting some "natural" expectations from a complexity ranking as systematic driver of sustainability in order perform the quality of our suggestion. One open question for us is: What are the additional coherency conditions that would be of interest and how can we make the list free of redundancy? From a theoretical point of view this seems to be very challenging question but might help to improve the quality of the ECI ranking and contribute to the transparency and communication.

- Class of coherent ranking measure for complexity and sustainability: We can support the fact that the second eigenvector is to a certain extent an eligible complexity measure due to its relationship with Shannon Entropy and coherent systematic driver of sustainability to a certain extend. However, as a systematic driver of sustainability, investigation of coherency of further alternatives could be an interesting research area that could have useful contribution to theory of economic sustainability.

- "Top down" internal complexity and internal sustainability ranking: Our main hypothesis is that the supplementary import trade information and its amalgamation with export data gives insight also about the internal economy. The question is: Given the supplementary export and import information matrices and their amalgamated version, is it possible to generate top-down internal complexity ranking as a systematic driver of internal sustainability from this ingredient? A positive answer to this question would deliver comparable internal ranking of countries.

- Empirical test of a sustainability index: In our opinion, resilience is a part of sustainability that refers to the ability to cope with challenging extreme events. Therefore, backtesting of a ranking based on extreme events (e.g.,) is an outlook that we can imagine. In this sense, depending on the availability of data, the back-testing of the quality of the amalgamated ECI based on the corona crisis is an interesting outlook.

\section{Concluding Remarks}

Countries that achieve economic complexity in a holistic way are well-prepared to respond to external shocks through internal processes that may also impact their future export structure and sustainability (in terms of resilience). It helps them to increase not just their economic complexity, but also their sustainability, and with it the ability to reconcile economic growth with environmental and social concerns. In other words, a country's future is more likely to be secured if its economic activities also contribute to its resilience, which requires an awareness about different possible scenarios and an ability to respond to such scenarios. Farsighted investments in sustainable and scalable innovation as well as the ability to pursue a plan B to effectively respond to socio-political, environmental, and financial shocks on a global as well as on a local scale contribute substantially to a country's resilience, which increases with the growth of economic complexity in a holistic way.

A complex and resilient economy is also the product of a successful collaboration between different actors largely operating in the domestic economy and the large, globally competitive export-oriented firms in the country in jointly responding to external trends and shocks by also exploiting the new opportunities that emerge from it. This ability to cooperate and with it to hold together the knowledge and knowhow that enables a complex 
economy to respond and enhance its resilience is built on bridging social capital. At the same time, the ability to create such products enhances the actor's social capital base and thus opens opportunities for favorable terms in future stakeholder collaboration. In other words, the acquisition of social capital through an outstanding performance helps to reduce the costs of maintaining the linkages within the domestic economic networks and makes it easier to build new ones.

This enhances the ability to respond to new regulation and scarcity through scalable innovation. Firms that operate within these networks are more likely to gain new projects and market shares, not merely through competition but also cooperation. These hybrid and evolving nodes of interactions among units within a particular domestic network develop organically and are firmly embedded in the wider economic system. For that purpose, grouping countries in accordance with their degree of resilience based on economic complexity information such as import data in addition to the standard global export information will have to be taken into account to a greater degree.

Our novel methodological approach applied to a limited but well-selected empirical investigation supports the hypothesis that the amalgamation of supplementary value chain information of import and export is of relevance to the improvement of the ECI or other alternative sustainability rankings. Our interpretation of the results and the coherency check supports that the ECI is a valuable measurement instrument for accumulated productive knowledge to a certain extent. Other ranking methods or even simple diagonal elements of the weighting matrix may offer an alternative as a coherent country ranking of sustainability, which behave well after amalgamation.

Complex methods may yield more concise results but at the expense of practicability whereas simple ones may be more practical but at the expense of the accuracy related to statistic and probabilistic interpretations of the empirical results. In summary, we can say that there is a tradeoff between theoretical perfection and pragmatism. Nevertheless, we think that comprehensive and deeper analyses of ECI and other alternatives by means of consolidated coherency conditions would contribute to deeper understanding of sustainability engineering and mechanics of future suggestions in this area.

Supplementary Materials: The manuscript containing all the mathematical treatments of the model and the extensive technical material is available online at https:/ / www.mdpi.com/2071-1050/13/4 /2049/s1.

Author Contributions: Conceptualization, B.C. and I.C.; methodology, B.C. and I.C.; data curation, B.C., I.C., and I.S.; validation, I.S., B.C., and I.C.; coding and numerical analysis B.C.; writingoriginal draft preparation, I.C.; writing-review and editing, I.S., P.A., and B.C. All authors have read and agreed to the published version of the manuscript.

Funding: This research received no external funding.

Institutional Review Board Statement: Not applicable.

Informed Consent Statement: Not applicable.

Data Availability Statement: The data presented in this study are available in "Amalgamation of Export with Import Information: The Economic Complexity Index as a Coherent Driver of Sustainability".

Acknowledgments: The authors would like to thank Eugenio Paris for his support in programming, numerical analysis and his availability as a sparring partner.

Conflicts of Interest: The authors declare no conflict of interest.

\section{References}

1. Hidalgo, C.A.; Hausmann, R. The building blocks of economic complexity. Proc. Natl. Acad. Sci. USA 2009, 106, 10570-10575. [CrossRef] [PubMed]

2. Hausmann, R.; Hidalgo, C.A.; Bustos, S.; Coscia, M.; Chung, S.; Jimenez, J.; Simoes, A.; Yıldırım, M.A. The Atlas of Economic Complexity-Mapping Paths to Prosperity; MIT Press: Cambridge, MA, USA, 2014; 368p.

3. Deardorff, A.V. The general validity of the Heckscher-Ohlin theorem. Am. Econ. Rev. 1982, 72, 683-694.

4. Buccellato, T. The Competences of Firms are the Backbone of Economic Complexity. SSRN 2016. [CrossRef] 
5. Aerni, P. Global Business in Local Culture: The Impact of Embedded Multinational Enterprises; Springer Briefs in Economics; Springer: Cham, Switzerland, 2018; 122p.

6. Jacobs, J. The Economy of Cities; Random House: New York, NY, USA, 1969; 268p.

7. Reynolds, C.; Agrawal, M.; Lee, I.; Zhan, C.; Li, J.; Taylor, P.; Mares, T.; Morison, J.; Angelakis, N.; Roos, G. A sub-national economic complexity analysis of Australia's states and territories. Reg. Stud. 2018, 52, 715-726. [CrossRef]

8. Chávez, J.C.; Mosqueda, M.T.; Gómez-Zaldívar, M. Economic complexity and regional growth performance: Evidence from the Mexican Economy. Rev. Reg. Stud. 2017, 47, 201-219.

9. Decker, M.; Fischer, M.; Ott, I. Service Robotics and Human Labor: A first technology assessment of substitution and cooperation. Robot. Auton. Syst. 2017, 87, 348-354. [CrossRef]

10. Carrillo-Tudela, C.; Gartner, H.; Kaas, L. Recruitment Policies, Job-Filling Rates and Matching Efficiency; IZA Discussion Paper No. 13240; IZA Institute of Labor Economics: Bonn, Germany, 2020; Available online: https:/ / papers.ssrn.com/sol3/papers.cfm? abstract_id=3602416 (accessed on 29 January 2021).

11. Hidalgo, C. Why Information Grows: The Evolution of Order, from Atoms to Economies; Basic Books: New York, NY, USA, 2015.

12. Brummitt, C.D.; Huremović, K.; Pin, P.; Bonds, M.H.; Vega-Redondo, F. Contagious disruptions and complexity traps in economic development. Nat. Hum. Behav. 2017, 1, 665-672. [CrossRef] [PubMed]

13. Trotter, H.F. On the product of semi-groups of operators. Proc. Am. Math. Soc. 1959, 10, 545-551. [CrossRef]

14. Chernoff, P.R. Product formulas, nonlinear semigroups, and addition of unbounded operators. Mem. Am. Math. Soc. 1974, 140, 1-121. [CrossRef]

15. Shen, A.; Uspensky, V.A.; Vereshchagin, N. Kolmogorov Complexity and Algorithmic Randomness; American Mathematical Society: Providence, RI, USA, 2017; Volume 220, 511p.

16. Atlas of Economic Complexity. Available online: https:/ /atlas.cid.harvard.edu/ (accessed on 29 November 2020).

17. World Bank Development Indicators. Available online: https://databank.worldbank.org/source/world-development-indicators (accessed on 29 November 2020).

18. Credit Suisse Global Wealth Databook 2019. Available online: https:/ /www.credit-suisse.com/about-us/en/reports-research/ global-wealth-report.html (accessed on 29 November 2020).

19. Shannon, C.E. A mathematical theory of communication. Bell Syst. Tech. J. 1948, 27, 623-656. [CrossRef]

20. Albeaik, S.; Kaltenberg, M.; Alsaleh, M.; Hidalgo, C.A. 729 new measures of economic complexity (Addendum to Improving the Economic Complexity Index). arXiv 2017, arXiv:1708.04107.

21. Dopfer, K.; Foster, J.; Potts, J. Micro-meso-macro. J. Evol. Econ. 2004, 14, 263-279. [CrossRef]

22. Haber, H.E. Notes on the Matrix Exponential and Logarithm; Santa Cruz Institute for Particle Physics, University of California: Santa Cruz, CA, USA, 2018; Available online: http:/ / scipp.ucsc.edu/ \{\}haber/webpage/MatrixExpLog.pdf (accessed on 22 January 2021). 